\section{Early termination of treatment in personality disorder treated in a psychotherapy hospital}

\author{
Quantitative and qualitative study ${ }^{\dagger}$
}

MARCO CHIESA, CARLA DRAHORAD and SILVIA LONGO

\author{
Background Factors underlying \\ premature discontinuation of psychosocial \\ in-patient treatment are still unclear.
}

\begin{abstract}
Aims Investigation of early discontinuation of specialised in-patient psychosocial treatment in a sample of people with personality disorder.
\end{abstract}

\begin{abstract}
Method Out of 134 consecutive admissions to the Cassel Hospital, 42 early drop-outs and 92 patients who remained were compared on demographic and clinical variables. Early drop-outs were invited for in-depth interviews, to explore their hospital experiences.
\end{abstract}

Results The early drop-out group and the group which remained showed significant differences in occupational status, borderline personality disorder (BPD) and the treatment programme to which they were allocated. All three independent variables predicted early discontinuation of treatment. The qualitative analysis of interview transcripts identified significant problems in institutional dimensions.

\section{Conclusions Important subjects and} process variables contributing to early drop-out in people with personality disorder were identified, with potential implications for clinical practice.

Declaration of interest This study was funded by a grant from the Sir Jules Thorn Charitable Trust.
It is universally recognised that the treatment of personality disorder is a difficult and challenging task. The severity of psychopathology, characterised by a mixture of low tolerance for frustration, frequent acting-out behaviour, chaotic patterns of relating, affective instability and high degree of passive aggression (Kernberg, 1975), makes treatment compliance notoriously difficult to obtain. In this study we analysed data from subjects with a diagnosis of personality disorder according to DSM-III-R criteria (American Psychiatric Association, 1987) who were consecutively admitted to the Adult Unit of the Cassel Hospital over a 5-year period. A combined quantitative and qualitative approach was used, in order to reach an in-depth understanding of the possible factors underlying early termination of treatment. First, we compared a sample of early drop-outs with a sample of patients who continued their treatment, on demographic and clinical variables. Second, we analysed transcripts of in-depth interviews with a subsample of early drop-out patients to identify problem areas in their experience while in hospital. In recent years attention has been paid to the advantages of combining quantitative and qualitative elements for a better, and complementary, understanding of phenomena under study (Mays \& Pope, 1996; Buston et al, 1998).

\section{Previous studies}

The literature on personality disorder shows high drop-out rates $(44-66 \%)$ in hospital-based treatments (Skodol et al, 1983; Gunderson et al, 1989; Kelly et al, 1992). These comparative studies found that attrition in borderline personality disorder (BPD) is significantly higher than in non-borderline personality disorder (NBDP), but some of their findings were contradictory. While Skodol et al (1983) found that drop-outs have had significantly more prior treatment, and had more severe symptoms at baseline, Gunderson et al (1989) and Kelly et al (1992) showed opposite findings in their samples.

Systematic analysis of data which takes into account the views of the users of the service is included in only three studies of generic psychotherapy out-patient populations (Acosta, 1980; Hynan, 1990; Pekarik, 1992). To our knowledge, attrition studies that include a qualitative analysis have not yet been carried out in specialised in-patient psychotherapy units. We feel that this is a considerable gap in the literature, and that more studies in this area are needed to achieve a better understanding of early drop-out.

\section{Hospital setting}

The Cassel Hospital is renowned for employing a psychodynamically based approach to the treatment and management of personality disorder in adults, adolescents and whole families. Medium-term residential treatment in in-patient psychotherapy units may be indicated for personality disorder for which previous general psychiatric and out-patient psychotherapeutic treatment has failed. The advantages of in-patient treatment include better compliance with treatment, fostering of the therapeutic alliance, a systematic challenge to destructive behaviour, and containment of suicidal gestures (Wallerstein, 1986; Norton \& Hinshelwood, 1996). The combination of a specific sociotherapeutic programme (Chapman, 1984), and individual twice-weekly psychotherapy delivered by medical and non-medical psychotherapists is the cornerstone of treatment at the Cassel. The hospital functions according to modified therapeutic community principles based on a 'culture of enquiry' (Main, 1989). Patients are admitted to two different programmes, based on geographical considerations: those residing within the Greater London Area (GLA) are allocated to a two-stage model (6 months in hospital followed by 18 months' psychosocial outreach work), while subjects residing outside the GLA are allocated to a one-stage model (1-year hospital stay, with no out-patient followup). Most patients admitted are on psychotropic medication; this is continually monitored and gradually withdrawn as treatment progresses.

Although some components of treatment are unique, the hospital programme shares core structural and cultural 
dimensions with other therapeutic communities and in-patient psychotherapy units. In these settings, the impact of high dropout rates is considerable for various reasons. First, there is evidence that the longer the patient stays in treatment the more likely they are to benefit from it (Waldinger \& Gunderson, 1984; Kelly et al, 1992). Second, the high demand made on health and social services (Chiesa et al, 1996; Dolan et al, 1996) by these patients would continue if specialised treatment failed. Third, the higher turnover resulting from patients terminating their treatment prematurely is considered to be unsettling for the stability of the milieu, the cohesion of the patients' group and their sense of belonging. A high incidence of early drop-out leads to low staff morale and a sense of worthlessness of the treatment offered, and to insecurity in the larger patients' community.

\section{METHOD}

\section{Definition of early drop-out}

Discontinuation of treatment is defined by a time frame that varies according to the expected length of treatment. In our study, early drop-out was defined as any premature termination of treatment, not mutually negotiated and agreed upon by staff and patient, that occurred within 14 weeks of admission. Previous findings indicate that early drop-outs do not benefit from psychosocial treatment (Hynan, 1990; Baruch et al, 1998).

\section{Study sample}

Out of 134 consecutive patients admitted to the Cassel Hospital from January 1993 to July 1997, 42 (32\%) left the hospital within 14 weeks. Only 14 patients (11\%) dropped out of treatment after 14 weeks (but before completion of the course), two $(1.5 \%)$ committed suicide while still in hospital and $76(57 \%)$ completed the course of treatment.

\section{Quantitative data collection}

As part of a prospective outcome study (Chiesa \& Fonagy, 2000) systematic information on all patients was obtained at intake, and at 6,12 and 24 months. The following instruments were used: the Structured Clinical Interview for DSM-III-R (Spitzer et al, 1990); the Symptom Checklist-90 (Derogatis, 1983); the Social Adjustment Scale (Weissman, 1975) and the
Global Assessment Scale (Endicott et al, 1976).

\section{Statistical analysis}

The statistical analyses were performed in SPSS for Windows (release 6.1). Chi-square was used to compare categorical variables between the early drop-out group and the group remaining; when the expected frequencies were below five, Fisher's exact test was used. Means were compared using the $t$-test for independent samples, except when distribution was not normal; then the Mann-Whitney $U$-test was performed. Logistic regression was used to determine the independent contribution of variables to early termination of treatment. Significance rested on a log likelihood test between the full model and one in which the main effect was removed.

\section{Qualitative data collection}

The 42 patients who left the hospital within 14 weeks were invited for a meeting with one of the researchers, to explore their experiences during their contact with the hospital. Nine patients refused the offer of a meeting, two were no longer alive, and nine could not be traced, while four declined to be interviewed on the grounds that they felt that they had little to contribute to the study. Eighteen patients $(43 \%)$ accepted the invitation to meet with one of two researchers (C.D. and S.L.).

The method used was one of semistructured in-depth interviews. The interviews were immediately dictated and subsequently transcribed. The three researchers subjected each transcription to content analysis, modelled on the method described by Miles \& Huberman (1994), which consists of highlighting sentence fragments that may indicate the presence of significant problem areas which the subject encountered during their stay at the hospital. Highlighted passages were subsequently organised according to problem area themes, which in turn were grouped into six general problem area categories. These categories represent a list of structural, cultural and process dimensions characteristic of the Cassel Hospital psychosocial approach. The transcripts were finally scored separately by two raters (C.D. and S.L.). An average $91 \%$ interrater agreement was reached.

\section{RESULTS}

\section{Quantitative analysis}

Univariate analysis showed a significant difference between early drop-outs and those remaining in level of occupation, borderline personality disorder status and the treatment programme to which they were allocated, and a nearly significant trend $(P<0.07)$ in educational status (Table 1). Patients who were employed in a skilled manual, partly skilled or unskilled occupation (the lower 3 levels) were significantly more likely to leave the hospital within 14 weeks of admission than those who held a higher and lesser professional or skilled non-manual occupation (top 3 levels) $\left(\chi^{2}=6.11, P<0.01\right)$. A significantly lower attrition rate was found in patients meeting criteria for borderline personality disorder (BPD) when compared with those not having borderline personality disorder (NBPD) $\left(\chi^{2}=6.91, P<0.01\right)$. Subjects allocated to the two-stage model of treatment showed a significantly lower early dropout rate than subjects in the one-stage programme $\left(\chi^{2}=4.73, P<0.03\right)$. A logistic regression model (Table 2 ) showed that all three terms in the equation (level of occupation, treatment allocation and borderline status) make a significant independent contribution to the prediction of early drop-out. The odds ratios of early drop-outs are 3.31 (95\% CI 1.36-8.03), 2.50 (95\% CI 1.01$6.25)$ and 2.50 (95\% CI 1.35-7.69) for the lower $v$. the higher levels of occupation, for the one-stage $v$. the two-stage subjects, and for NBPD $v$. BPD respectively. Contingency tables showed that $36 \%(n=17)$ of those with BPD in the one-stage programme dropped out, compared with only $8 \%(n=3)$ in the two-stage programme. In the logistic regression model, a significant treatment allocation by BPD interaction was found $(\beta=-1.72$, s.e. $=0.88$, d.f. $=1$, $P<0.05)$. The odds ratios of early dropout show that those with BPD in the onestage programme are 5.5 (95\% CI 0.99 31.20 ) times more likely to drop out early in treatment than those with BPD in the two-stage programme.

\section{Qualitative analysis}

The analysis of the 18 available transcripts resulted in 50 positive scores on the area categories experienced as problematic (Table 3). Of these, $41(82 \%)$ applied to three categories: institutional culture and structure, organisation of treatment and 
Table I Comparison between early drop-outs and those continuing treatment on demographic and clinical variables

\begin{tabular}{lcc}
\hline Independent variables & Early drop-outs, $n=42$ & Continuers, $n=92$ \\
\hline Age: mean (s.d.) & $33(8.1)$ & 31 (7.5) \\
Gender (female): $n$ (\%) & $31(73.8)$ & $71(77.2)$ \\
Education (above GCSEs): $n$ (\%) & $23(54.8)$ & $65(70.7)$ \\
Occupation (lower 3)*: $n$ (\%) & $20(52.6)$ & $21(28.8)$ \\
Symptom Checklist-90': mean (s.d.) & $1.92(0.69)$ & $1.91(0.77)$ \\
Social Adjustment Scale': mean (s.d.) & $2.64(0.49)$ & $2.65(0.51)$ \\
Serious suicide effort: $n$ (\%) & $7(16.7)$ & $9(9.8)$ \\
Drug \& alcohol abuse: $n$ (\%) & $14(31.1)$ & $31(68.9)$ \\
Externally directed aggression: $n$ (\%) & $27(30.0)$ & $9(21.4)$ \\
Length of previous hospitalisation (days): mean (s.d.) & $60.8(85.5)$ & $49.7(79.5)$ \\
Number of previous hospitalisations: mean (s.d.) & $1.2(1.9)$ & $0.7(0.9)$ \\
Time on medication (weeks): mean (s.d.) & $8.1(5.1)$ & $8(5.1)$ \\
Number of personality disorders: mean (s.d.) & $3.65(1.69)$ & $3.39(1.82)$ \\
Borderline personality disorder**: $n$ (\%) & $22(44.0)$ & $18(22.2)$ \\
Treatment allocation* & & $47(78.3)$ \\
$\quad$ One-stage programme: $n$ (\%) & $29(39.2)$ & $13(21.7)$ \\
$\quad$ Two-stage programme: $n$ (\%) & & $45(60.8)$ \\
\hline
\end{tabular}

$* P<0.03, * * P<0.01$

I. Derogatis (1983)

2. Weissman (1975)

Table 2 Logistic regression predicting early drop-out $v$. continuation of treatment

\begin{tabular}{lccc}
\hline Independent variable & $\begin{array}{c}\text { Standardised coefficient } \\
(\beta)\end{array}$ & $\begin{array}{c}\text { s.e. of standardised } \\
\text { coefficient }\end{array}$ & $\chi^{2}$, d.f.=I \\
\hline Level of occupation & 1.197 & 0.453 & $7.28^{*}$ \\
Borderline personality disorder & -1.192 & 0.452 & $7.30^{*}$ \\
Treatment allocation & -0.914 & 0.465 & $4.1 I^{* *}$ \\
Treatment allocation by BPD & -1.72 & 0.882 & $4.10^{* *}$ \\
\hline
\end{tabular}

$* P<0.05, * * P<0.01$.

relationship with other patients. The main problems as perceived by patients can be summarised as follows.

\section{Cultural and structural set-up and general treatment approach}

(a) Staff misunderstand patients' behaviour. Specifically, staff fail to appreciate the fears and vulnerabilities behind acting-out, aggressive behaviour and alcohol-related problems, which are approached in a moralistic and punitive fashion.

(b) The decision-making processes do not take patients' views sufficiently into account. Hospital staff 'hide behind their role' and are difficult to reach on a human level. (c) Staff expect too much too soon from newly admitted patients, who are not given enough time to settle and get used to the pace of community life.

\section{Treatment organisation and delivery}

(a) Community life is incompatible with the need for privacy. Attempts to seek individual space are 'frowned upon' and challenged as a resistance to full involvement in treatment.

(b) Participation in hospital activities is felt as a compulsory duty, rather than a therapeutic tool.

(c) Small and large group meetings become all too often persecutory.

(d) Treatment delivery is not sufficiently flexible. Patients 'are not treated as individuals' with different backgrounds and individual needs, but are treated in a 'standardised way'.

(e) The treatment approach and philosophy are not made sufficiently clear at admission; patients do not know 'what to expect in therapy sessions or group meetings'.

Dimensions of living together in a therapeutic community

(a) Too much responsibility is placed on some patients to support acting-out patients. They become like 'emotional sponges soaking up all the suicidal depression around them'.

(b) An anti-therapeutic patients' subculture was reported, including intimidation, bullying and backbiting from a dominant subgroup of patients.

\section{DISCUSSION}

The results from our sample show that a higher occupational status, having a borderline diagnosis and belonging to the two-stage programme are predictors of continuation of treatment. Education also shows a trend towards significance. Possibly, better educational and occupational status equips patients with greater resilience to withstand difficulties in the short term, in the hope of later positive results. This finding is consistent with Garfield's review (1994), which identified social class variables as most consistently correlated with continuation in psychotherapy.

\section{Borderline personality disorder and early drop-out}

The superior in-patient treatment compliance showed by subjects with borderline personality disorder in the two-stage programme is an interesting finding. Since geographical factors and differences in the two populations have been excluded in two recent studies (Chiesa, 1997; Chiesa \& Fonagy, 2000), considerations of the treatment model may provide a possible explanation. A two-phase model may allow a better working-through of emotionally laden conflicts to do with termination and separation from treatment, which is established as a crucial feature in the treatment of BPD. The prospect of a shorter in-patient stay and an assured longer-term continuation of treatment as an outpatient may render more tolerable the claustrophobic and persecutory anxieties 
Table 3 Qualitative analysis of in-depth interviews with drop-out sample $(n=18)$

\begin{tabular}{|c|c|c|}
\hline Problem area categories & Problem area themes & Total score \\
\hline $\begin{array}{l}\text { Institutional culture and structure (overall } \\
\text { treatment approach and philosophy of } \\
\text { treatment) }\end{array}$ & $\begin{array}{l}\text { Not feeling understood } \\
\text { Undemocratic attitude } \\
\text { Premature high expectations } \\
\text { Rigid attitudes, lack of flexibility } \\
\text { Separation from the outside world } \\
\text { Overconfrontative attitude } \\
\text { Overanalytical behaviour }\end{array}$ & 16 \\
\hline $\begin{array}{l}\text { Organisation of treatment (how the treatment } \\
\text { is structurally delivered on a day-to-day basis) }\end{array}$ & $\begin{array}{l}\text { Time management } \\
\text { Domestic chores } \\
\text { Usefulness of group activities } \\
\text { Too much responsibility } \\
\text { Allocation to treatment programme } \\
\text { Accommodation arrangements }\end{array}$ & 14 \\
\hline $\begin{array}{l}\text { Relationship with other patients (living together, } \\
\text { and patients' subculture) }\end{array}$ & $\begin{array}{l}\text { Other patients' disturbance } \\
\text { Lack of private space } \\
\text { Scapegoating and backbiting } \\
\text { Exclusion from the main group }\end{array}$ & II \\
\hline Relationship with key nurse & $\begin{array}{l}\text { Not sufficiently available } \\
\text { Lack of confidentiality }\end{array}$ & 4 \\
\hline Relationship with therapist & $\begin{array}{l}\text { Therapist's gender } \\
\text { Lack of trust } \\
\text { Lack of confidentiality }\end{array}$ & 4 \\
\hline Other & Fear of research programme & 1 \\
\hline
\end{tabular}

stirred by the intensely challenging and confrontational nature of the hospital psychosocial approach.

\section{Clinical implications of qualitative analysis}

The results from the analysis of the patients' experience of the hospital setting may have implications for clinical practice in therapeutic community settings. Most of the problem areas identified implicated the dimensions of the prevailing institutional culture, the way treatment was delivered and aspects of the patients' living together. A too uniform application of the treatment programme, which does not take into account individual differences, a rigidity in applying the rules and an excessive confrontational attitude at the expense of containment and understanding, constitute the main findings. This emerging pattern seems opposed to the declared hospital philosophy of treatment, which stresses understanding, enquiry into patient and staff dynamics, flexibility, constant dialogue and creative thinking. In therapeutic community and group settings, the coexistence of an unacknowledged (hence difficult to modify) in treatment. A change of focus may be needed, in the direction of reviewing and improving treatment structures, and the cultural attitudes and expectations of the staff.

\section{Methodological considerations}

Although we feel that this study makes an original contribution to the literature on early discontinuation of psychotherapeutic treatment, some limitations ought to be borne in mind when interpreting the results. First, the study was carried out on a selected sample of individuals with personality disorder admitted to a specialised in-patient setting from a wide geographical area. Although other therapeutic communities, which share a similar treatment philosophy and modality, may benefit from the results of this study, the generalisability of the findings to wider settings is limited.

Qualitative results are based on indepth interviews conducted with a drop-out sample. To obtain a more comprehensive picture, data on the hospital experiences of the group remaining in hospital, for comparison with those of the drop-out group, are also needed. Preliminary results from a survey of treatment satisfaction of those who remained shows a significant degree of convergence of findings between them and the drop-outs. These results make a strong argument in favour of initiating a process of revision of the structural, cultural and organisational set-up of the hospital.

\section{ACKNOWLEDGEMENTS}

Sophia Rabe-Hesketh, PhD, provided help with statistical analysis; and we are grateful to the patients who agreed to be interviewed.

\section{REFERENCES}

Acosta, F. X. (1980) Self described reasons for premature termination of psychotherapy by Mexican American, Black American, and Anglo-American patients. Psychological Reports, 47, 435-443.

American Psychiatric Association (1987) Diagnostic and Statistical Manual of Mental Disorders (3rd edn, revised) (DSM-III-R). Washington, DC: APA

Baruch, F., Gerber, A. \& Fearon, P. (1998)

Adolescents who drop out of psychotherapy at a community based psychotherapy centre: a preliminary investigation of the characteristics of early drop-outs, late drop-outs and those who continue treatment. British Journal of Medical Psychology, 7I, 233-245.

Buston, K., Parry-Jones, W., Livingstone, M., et al (1998) Qualitative research. British Journal of Psychiatry, I72, 197-199. 
Chapman, C. E. (1984) A therapeutic community, psychosocial nursing and the nursing process. International Journal of Therapeutic Communities, 5, 68-76.

Chiesa, M. (1990) Subtle aspects of mental manipulation within the therapeutic community culture In La Persuasione Socialmente Accettata, il Plagio e il Lavaggio del Cervello (ed. M. Di Fiorino), pp. 160-165 Lucca: Psichiatria e Territorio.

_ (1997) A combined inpatient/outpatient programme for severe personality disorders. Therapeutic Communities, 18, 297-309.

_ , lacoponi, E. \& Morris, M. (1996) Changes in health service utilisation by patients with severe personality disorders before and after in-patient psychosocial treatment. British Journal of Psychotherapy, 12, 50I-512.

— \& Fonagy, P. (2000) Cassel Personality Disorder Study. Methodology and treatment effects. British Journal of Psychiatry, 176, 485-491.

Derogatis, L. R. (1983) SCL-90-R. Administration, Scoring and Procedures. Manual - II. Towson, MD: Clinical Psychometric Research.

Dolan, B. M., Warren, F. M., Menzies, D., et al (1996) Cost-offset following specialist treatment of severe personality disorders. Psychiatric Bulletin, 20, 413-4I7.

Endicott, J., Spitzer, R. L., Fleiss, J. L., et al (1976) The Global Assessment Scale. Archives of General Psychiatry, 33, 766-771.

Garfield, S. L. (1994) Research on client variables in psychotherapy. In Handbook of Psychotherapy and Behavior Change (4th edn) (eds A. E. Bergin \& S. L. Garfield), pp. 190-228. New York: Wiley.

Gunderson, J. G., Frank, A. F., Ronningstam, E. F., et al (1989) Early discontinuance of borderline patients from psychotherapy. Journal of Nervous \& Mental Disease, 177, 38-42.

Hynan, D. G. (1990) Client reasons and experiences in treatment that influence termination of psychotherapy. Journal of Clinical Psychology, 46, 891-895.

James, O. (1984) The role of nurse-therapist relationship in the therapeutic community. International Review of Psychoanalysis, II, 151-159.

Kelly, T., Soloff, P. H., Cornelius, J., et al (1992) Can we study (treat) borderline patients? Attrition from research and open treatment. Journal of Personality Disorders, 6, 417-433.

Kernberg, O. F. (1975) Borderline Conditions and Pathological Narcissism. New York: Aronson.

Main, T. F. (1967) Learning, knowledge and freedom from thought. Australian \& New Zealand journal of Psychiatry, I, 64-7I.

- (1989) The Ailment and Other Psychoanalytic Essays. London: Free Association Press.

Mays, N. \& Pope, C. (1996) Qualitative Research in Health Care. London: BMJ Publishing.

\section{CLINICAL IMPLICATIONS}

- A combined in-patient and out-patient model improves treatment compliance in borderline personality disorder.

- Ongoing critical enquiry into the cultural aspects of the in-patient setting, into the organisation of treatment delivery and into the subculture of the patients' group is required to improve the proportion of those who continue their treatment in a therapeutic community setting.

- The results may be relevant for other hospital-based settings concerned with personality disorder treatment and therapeutic communities.

\section{LIMITATIONS}

- Generalisability of results to a general psychiatric setting is limited.

- The qualitative part of the study needs to be extended to include a group of the patients who did not drop out, in order to reach more robust conclusions to guide clinical practice.

- The qualitative analysis is based only on the patients' viewpoint.

MARCO CHIESA, MRCPsych, CARLA DRAHORAD, DPsychol(Padua), SILVIA LONGO, DPsychol(Padua), Research Unit, The Cassel Hospital, Richmond, Surrey

Correspondence: Dr M. Chiesa, Research Unit, The Cassel Hospital, I Ham Common, Richmond, Surrey TWI0 7JF. e-mail: marco@cassel.ftech.co.uk

(First received 18 December 1998, final revision 18 October 1999, accepted 18 October 1999)

Miles, M. B. \& Huberman, A. M. (1994) Qualitative Data Analysis: An Expanded Sourcebook. Thousand Oaks, CA: Sage.

Norton, K. \& Hinshelwood, R. D. (1996) Severe personality disorder. Treatment issues and selection for in-patient psychotherapy. British Journal of Psychiatry, 168, 723-731.

Pekarik, G. (1992) Relationship of clients' reasons for dropping out of treatment to outcome and satisfaction. Journal of Clinical Psychology, 48, 91-98.

Skodol, A. E., Buckley, P. \& Charles, E. (1983) Is there a characteristic pattern to the treatment history of clinic outpatients with borderline personality? Journal of Nervous \& Mental Disease, $7 \mathbf{I}$ 405-410.

Spitzer, R. L., Williams, J., Gibbon, M., et al (1990) Structured Clinical Interview for DSM-III-R. Washington, DC: American Psychiatric Association.

Waldinger, R. \& Gunderson, J. (1984) Completed psychotherapies with borderline patients. American Journal of Psychotherapy, 38, 190-202.

Wallerstein, R. S. (1986) Forty-two Lives in Treatment: A Study of Psychoanalysis and Psychotherapies. New York: Guilford.

Weissman, M. M. (1975) The assessment of social adjustment. Archives of General Psychiatry, 32, 357-365. 